\title{
Fallstudie Swisscom:
}

«Die Einführung von

Produktions- und

Servicekatalogen -

Der erste Schritt zur

industrialisierten Fertigung»

Andreas Hilber, Denisa Kykalová und Elke Brucker-Kley

8.1 Kontext und Ausgangssituation - 112

8.2 Motivation und Zielsetzung - 113

8.3 Umsetzung und Wirkung - 116

$8.4 \quad$ Fazit -120

Literatur - 124 
Die vorliegende Fallstudie zeigt das Vorgehen und die Erfahrungen der Swisscom Network \& IT Operations bei der Standardisierung ihrer Leistungen und der Entwicklung einer neuen Service- und Produktarchitektur. Getrieben durch die steigende Komplexität der Produkte und die wachsenden Qualitätsanforderungen der Kunden galt es, bei gleichzeitiger Erhöhung der Qualität, Kosten zu reduzieren und die Effizienz zu steigern. Inspiration holte sich Swisscom aus der herstellenden Industrie und orientierte sich an der individualisierten Massenproduktion (industrialisierten Fertigung).

\subsection{Kontext und Ausgangssituation}

Swisscom ist das führende Telekommunikationsunternehmen und eines der führenden ITUnternehmen der Schweiz und bietet Geschäfts- und Privatkunden Mobilfunk, Festnetz, Internet, Digital-TV und Informatik-Dienstleistungen an. Über den Wholesale-Kanal stellt sie auch Dritten ihre Netz- und IT-Infrastruktur zur Verfügung. Mit 21.600 Mitarbeitenden erwirtschaftete Swisscom 2015 einen Jahresumsatz von rund CHF 11,6 Mia.

Das strategische Ziel der Swisscom - «die beste Begleiterin und vertrauensvolle und inspirierende Partnerin in der vernetzten Welt zu sein» und ihren Kunden immer, überall, sicher und einfach verfügbare Leistungen mit ausserordentlichen Erlebnissen zu bieten (vgl. - Abb. 8.1) - erfordert, dass sie gesellschaftliche, technologische und wirtschaftliche Trends antizipiert und aufnimmt. Als wegweisend für die Zukunft der ICT-Branche schätzt sie dabei die folgenden Trends ein (Swisscom AG 2015):

- Immer online: Die Digitalisierung führt dazu, dass nicht nur Menschen, sondern auch intelligente Applikationen und Geräte zunehmend miteinander vernetzt sein werden.

- Internetbasiert «All IP»: Produkte \& Services werden künftig auf Basis des Internet-Protokolls betrieben. Speicherplatz, Rechenleistung und Software werden verstärkt aus dem Internet bezogen.

- Globaler Wettbewerb: Weltweit tätige Mitbewerber profitieren von globalen Skaleneffekten und verändern die Geschäftsmodelle durch eine verstärkte Nutzung von Kundendaten.

Eine diesen Anforderungen entsprechende Netz- und IT-Infrastruktur, welche effizient und zielgerichtet eingesetzt werden kann, ist dabei eine Grundvoraussetzung. Sie muss nicht nur professionell unterhalten und betrieben, sondern auch kontinuierlich ausgebaut und immer wieder neu ausgerichtet werden, um dem wachsenden Bedarf an Leistungen gerecht zu werden: Der Breitbandbedarf im Festnetz verdoppelt sich alle 16 Monate, im Mobilfunk jährlich. Mit neuen Servicedienstleistungen steigt ebenfalls der Bedarf an Datacenterleistungen stetig an.

Doch technologischer Wandel, intensiver lokaler und globaler Wettbewerb und die sich verändernden Kundenbedürfnisse führen dazu, dass die Preise und Volumen im klassischen Geschäft mit nutzungsabhängigen Angeboten kontinuierlich erodieren. Trotzdem müssen hohe Investitionen in die sich verändernde Technologie und den Ausbau der Infrastruktur getätigt werden. Um dieser Entwicklung gerecht zu werden, muss einem Ergebnisrückgang auch durch Steigerung der Effizienz begegnet werden.

Vor diesem Hintergrund beschäftigte sich die Organisationseinheit «Network \& IT Operations» (ca. 2300 Mitarbeiter) damit, wie sie künftig ihren Auftrag - den zentralen Betrieb der Infrastruktur - effizient und günstig gestalten, und gleichzeitig die vereinbarte Qualität erhöhen kann. - Abb. 8.2 visualisiert die Aufgabe von «Network \& IT Operations» innerhalb des Prozessmodells von Swisscom. 


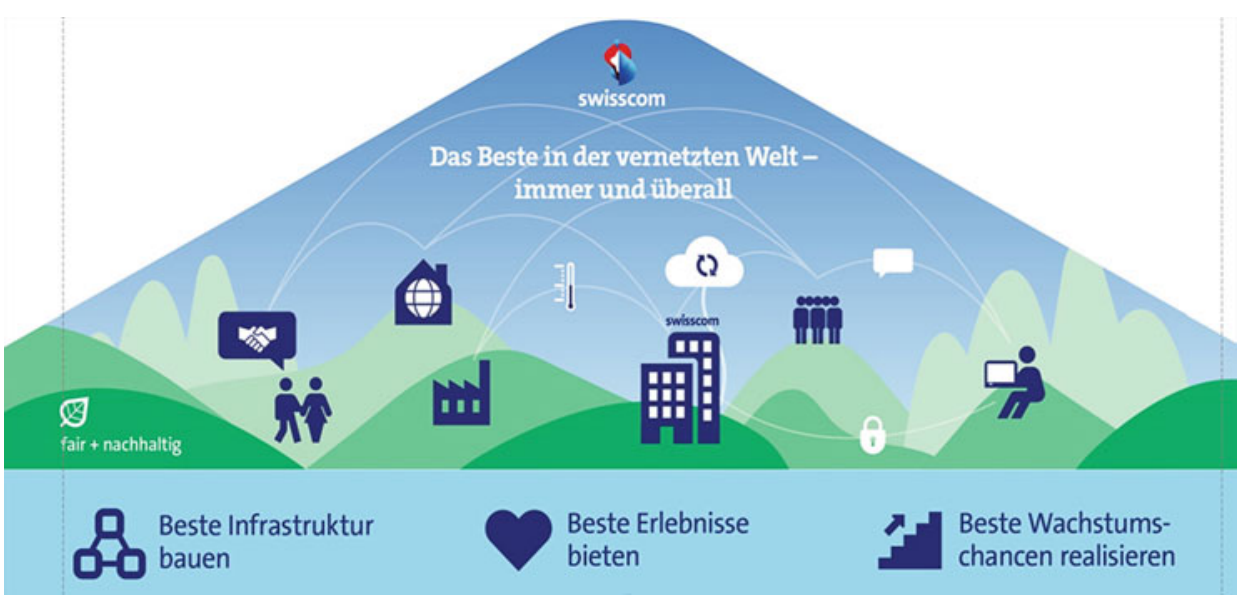

- Abb. 8.1 Unternehmensstrategie «Swisscom 2020«

\section{Prozessmodell Swisscom:}

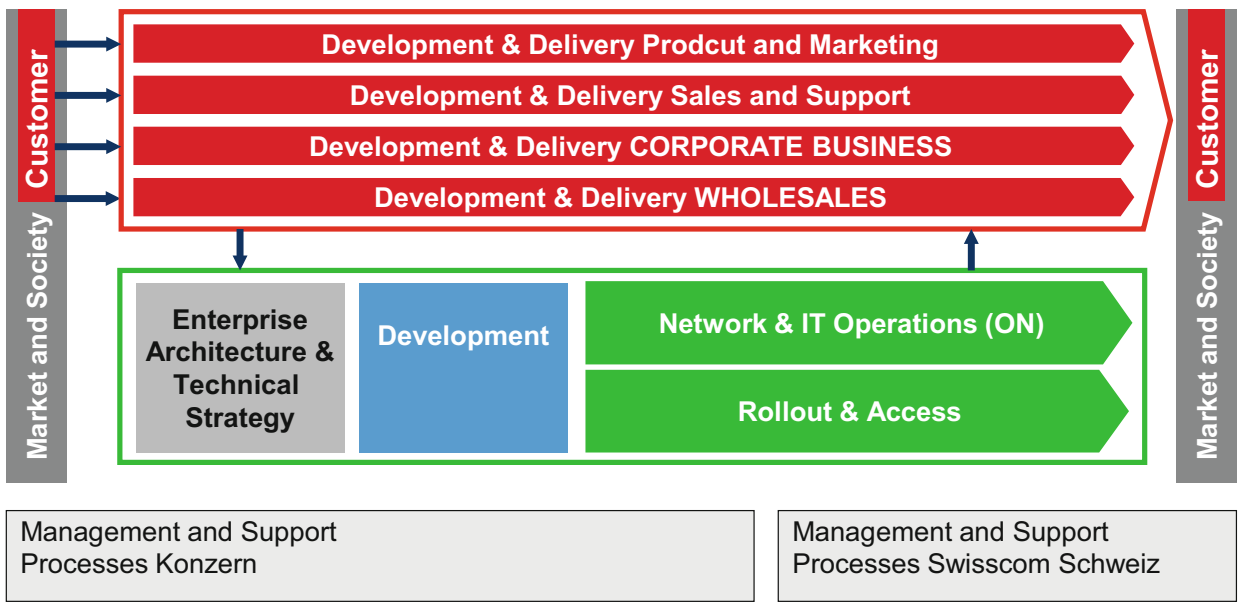

Legende:

Selling Services

Innovation

Production

Management \& Support Processes

- Abb. 8.2 Rolle der Organisationseinheit «Network \& IT Operations» im Prozessmodell Swisscom

\subsection{Motivation und Zielsetzung}

Durch den Zusammenschluss Swisscom IT Services und Swisscom Schweiz im Jahre 2014 wurde die Produktion in einer Einheit zusammengeführt, welche aufgrund ihrer Historien unterschiedliche Kundensegmente bediente und zum Teil überlappende Produktionskataloge 


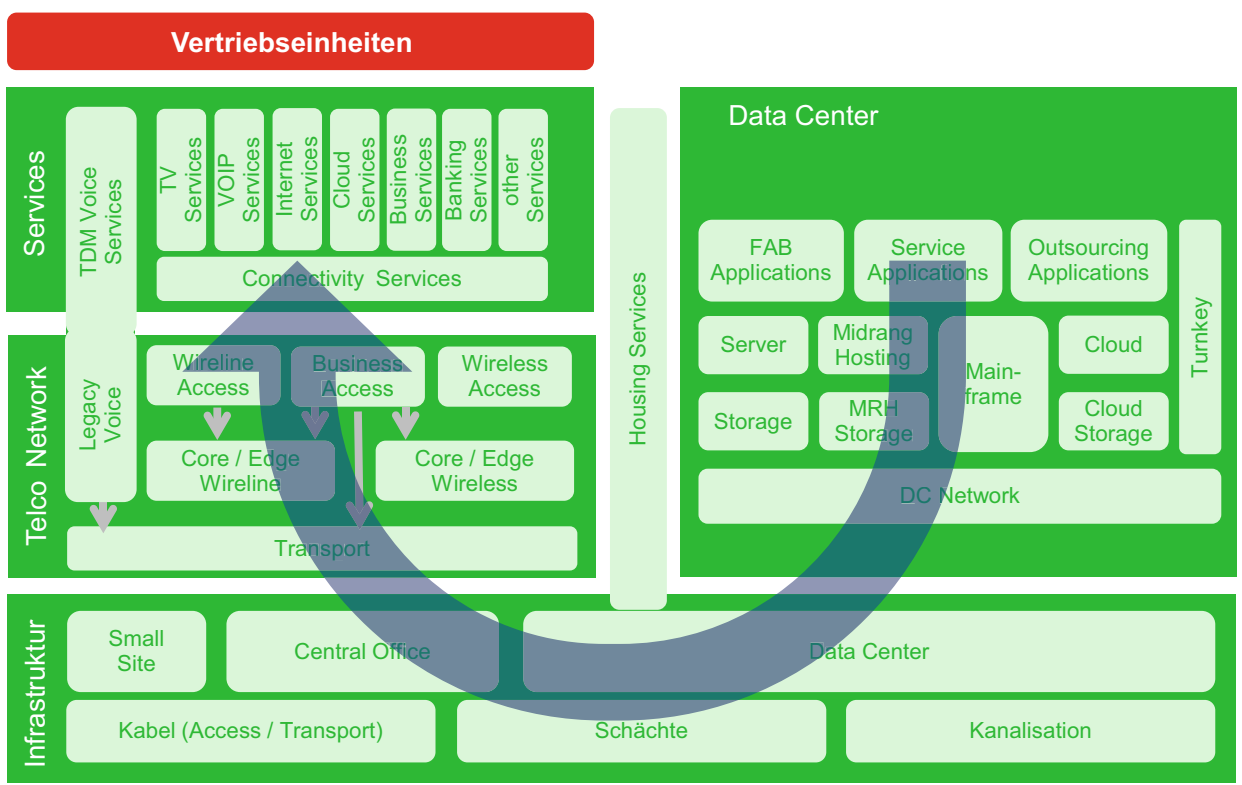

- Abb. 8.3 Fertigung in verteilten Fertigungsstrassen über viele OEs hinweg

aufwies. Ein einheitlicher gemeinsamer Katalog wurde als Hebel für die Standardisierung, Zusammenführung und Abstimmung der Leistungserbringung aufeinander erkannt (- Abb. 8.3). Dies mit dem Ziel, die Leistungs-, Qualitäts- \& Betriebskosten-Transparenz zu adressieren und auf die künftigen Herausforderungen optimal vorbereitet und ausgerichtet zu sein.

Mit dem katalogbasierten Ansatz soll sinngemäss eine Verschiebung von historisch entstandenen und dokumentierten Fertigungsprodukten hin zu strukturierten Fertigungsprodukten und industrialisierter Fertigung stattfinden ( $\bullet$ Tab. 8.1).

Gleichzeitig darf die Kundenperspektive nicht aus dem Fokus geraten, damit der Kunde seine Bedürfnisse in seiner eigenen Sprache formulieren kann, ohne sich zuerst mit dem technischen Hintergrund der Produkte und der dafür notwendigen Infrastruktur auseinandersetzen zu müssen.

I) Die Kunden wollen ein Service-Erlebnis und keine IT-Komponenten! (Andreas Hilber, Head of Process Architecture IT \& Network Operation, Swisscom AG)

- Tab. 8.1 Eigenschaften der industriellen Fertigung

\begin{tabular}{|c|c|c|}
\hline VON & & $\mathbf{Z U}$ \\
\hline $\begin{array}{l}\text { Historisch entstandene und dokumentier- } \\
\text { te Fertigungsprodukte }\end{array}$ & $\rightarrow$ & $\begin{array}{l}\text { Strukturierte Fertigungsprodukte und indus- } \\
\text { trialisierte Fertigung }\end{array}$ \\
\hline Hohe Komplexität & $\rightarrow$ & Funktionale, generische Merkmale \\
\hline Siloartiger Aufbau & $\rightarrow$ & Konfigurierbare Modularität \\
\hline Hohe Integrationskosten & $\rightarrow$ & Reduzierte Komplexität \\
\hline Toolabhängigkeit & $\rightarrow$ & Toolunabhängigkeit \\
\hline
\end{tabular}


- Abb. 8.4 Kernprinzipien der industriellen Fertigung, Trennung vom Was und Wie

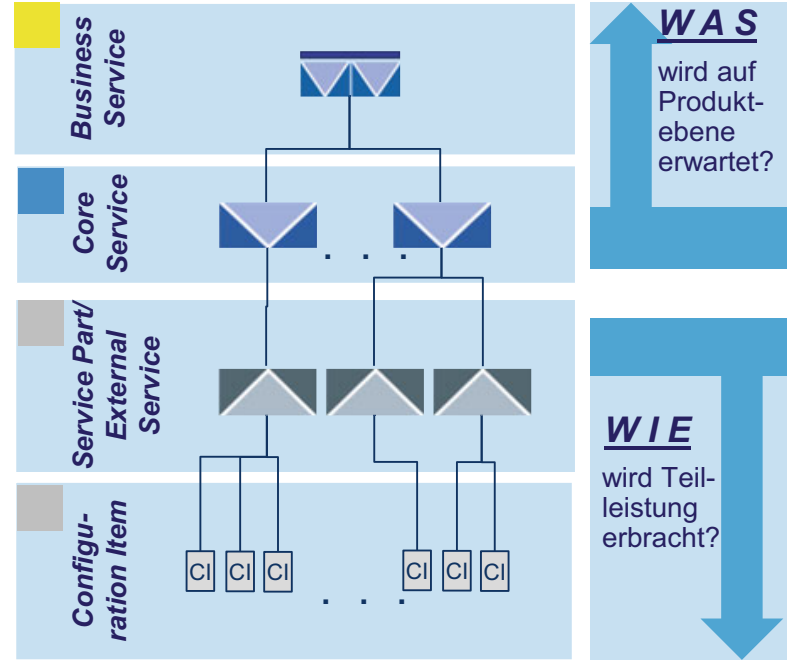

Die konsequente Trennung des WAS (Kundenwunsch) und des WIE (für Kunden Lösung erbringen) ist deshalb eines der Kernprinzipien, um die industrialisierte Fertigung umsetzen zu können. Erst ihre Befolgung erlaubt eine konsequente Standardisierung und damit Economy of Scale zu liefern bei gleichzeitiger Ermöglichung von Economy of Scope. Mit anderen Worten, Modularisierung der Leistungserbringung bei gleichzeitiger Standardisierung der Variabilität (Anzahl der Ausprägungen eines einzelnen Moduls).

\section{Industrialisierte Fertigung \\ Industrialisierung ist ein volkswirtschaftlicher Prozess, der durch signifikante Zunahme von gewerblicher Gütererzeugung mit wachsendem Maschineneinsatz in grossgewerblicher, arbeitsteiliger Produktionsorganisation geprägt ist (Springer Gabler, o.J.). \\ Durch die Fertigung hoher Güterstückzahlen können verschiedene Rationalisierungsmög- lichkeiten genutzt und tiefe Stückkosten erzielt werden. Wegen der wegfallenden häufigen Umrüstungsnotwendigkeit der Produktionsanlagen bietet sich eine Teil-/Automatisierung des Produktionsprozesses an.}

- Abb. 8.4 demonstriert die Mehrstufigkeit dieser Trennung von WAS und WIE. Ein Core Service ist die kleinste bestell-, liefer- und verkaufbare Leistungseinheit. Vordefinierte Konfigurationsmöglichkeiten und Optionen erlauben eine gewisse Individualisierung. Die Qualität wird in einem SLA vereinbart. Ein Service Part ist ein Halbfabrikat, das in einer Fertigungsstrasse produziert und in verschiedenen Core Services wiederverwendet werden kann. Er umfasst personelle Leistungen (Arbeitsaufträge, Prozesse, Stunden) und techn. Leistungen (HW, SW, Lizenzen etc.). Die Qualität wird in einem OLA definiert.

Von einem solchen Vorgehen verspricht sich Network \& IT Operations folgende operative Zielsetzungen zu erreichen und dadurch der Koexistenz zwischen Economy of Scale und Economy of Scope optimal begegnen zu können:

- Economy of Scope: Dank Modularisierung der Produkte wird der Swisscom-Vertrieb befähigt, die Endkunden flexibler, schneller und effizienter zu bedienen und somit Time-to- 
Market bei erhöhter Qualität zu senken (Verbundeffekte durch eine gemeinsam genutzte Infrastruktur der modularisierten Produkte ermöglichen eine optimierte Servicebereitstellung beim Kunden).

- Economy of Scale: Reduzierte Produktvariabilität ermöglicht Massenproduktion, Prozessstandardisierung und -automatisierung und eine bessere Steuerung der Betriebskosten. Dies resultiert in reduzierten Kosten und gesteigerter Effizienz.

॥ Unser Anspruch ist eine industrialisierte Fertigung in einer verteilten ICT-Produktionsumgebung zu schaffen, um damit einen Beitrag zu leisten, den Umsatz- und Ergebnisrückgang durch Steigerung der Effizienz kompensieren zu können. Und dies bei Erhöhung der zur Verfügung gestellten Qualität (Andreas Hilber, Head of Process Architecture, Network \& IT Operations, Swisscom AG).

\subsection{Umsetzung und Wirkung}

- In 5 Stufen zur industrialisierten Fertigung

Anfangs 2015 (Januar bis März) konzipierte Swisscom Network \& IT Operations ein fünfstufiges Vorgehenskonzept (- Abb. 8.5), welches sie zur industrialisierten Fertigung führen soll. Jede Stufe baut auf der vorangehenden Stufe auf, wobei jede Stufe für sich bereits einen Mehrwert erbringt.

1. Produktionskataloge für interne Betriebs- \& externe Markt-Leistungen:

Auf der untersten Stufe wird durch Dekomposition und Katalogisierung der produzierten Services in einer neuen Service- und Produktarchitektur der Grundstein zur industrialisierten Fertigung gelegt. Die neuen Kataloge sollen zunächst die IST-Situation spiegeln und die internen Produkte (Halbfabrikate) mit neuen Informationen (wie z. B. Herstellungskosten, Konfigurationsdaten, KPI-Zielwerte, Arbeitspläne (Prozess), Stücklisten (HW, SW ... )) angereichert werden. Diese müssen einheitlich erhoben und dargestellt werden, so dass normalisierte Daten entstehen und auf den höheren Stufen weiter genutzt werden können.

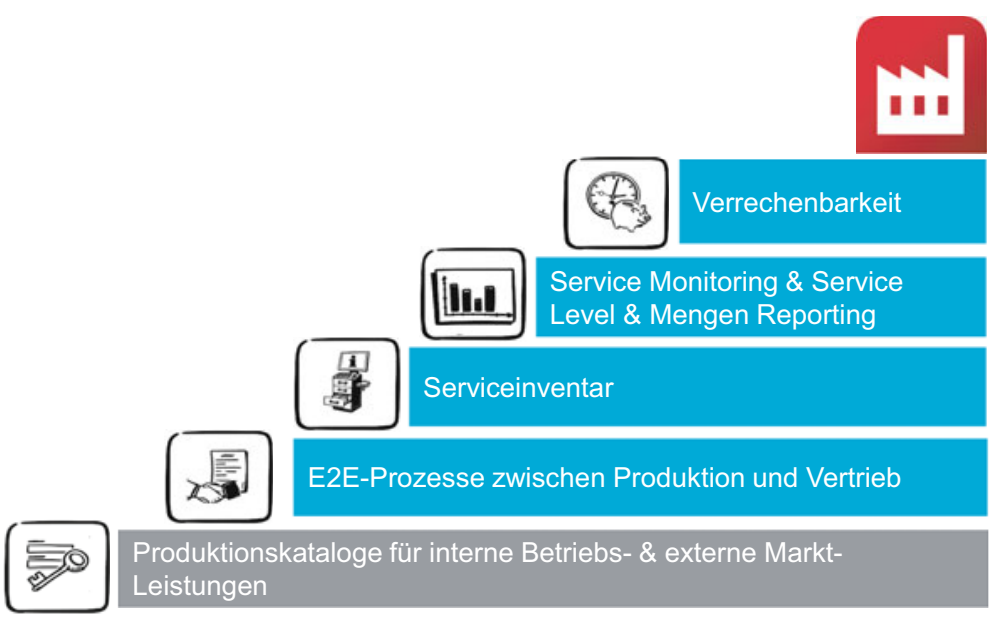

- Abb. 8.5 Die 5 Stufen zur industrialisierten Fertigung 
2. E2E-Prozesse zwischen Produktion und Vertrieb:

Auf der zweiten Stufe werden die modularisierten und katalogisierten Halbfabrikate aus der Produktion den Verkaufseinheiten zur Verfügung gestellt. Die Durchgängigkeit der Prozesse und die einfachere Handhabbarkeit der informationsreichen Kataloge sollen eine intensive Nutzung der Kataloge an der Front bewirken, was sich wiederum in planbaren Auslieferungs- und Herstellungsprozessen und einer kürzeren Time-to-Market niederschlägt.

3. Serviceinventar:

Auf der dritten Stufe sollen die effektiv laufenden Service-Instanzen gemäss Verträgen in einem Serviceinventar festgehalten werden, um einerseits ein konsistentes Inventar der Konfigurationselemente (CI, configuration item) über den Product Life Cycle entwickeln und benötigte Anpassungen der Infrastruktur erkennen zu können. Andererseits ermöglicht ein Serviceinventar auch eine kritische Auseinandersetzung mit dem bestehenden Produktangebot und seiner Neuausrichtung.

4. Service Monitoring \& Service Level und Mengen-Reporting: Ein proaktives Service Level Management hilft, die Herausforderungen der verteilten Produktionsumgebung zu managen.

5. Verrechenbarkeit:

Die durch die Umsetzung der unteren Stufen entstandene Kostentransparenz ermöglicht weitere Effizienzsteigerung.

- Phase 1

Im April 2015 begann Swisscom Network \& IT Operations die unterste Stufe des Vorgehensmodells «Produktionskataloge für interne Betriebs- \& externe Markt-Leistungen» in einer ersten Phase umzusetzen. Innerhalb von 10 Monaten wurde die gesamte Breite des Produktionsportfolios wie z. B. Connectivity, Grundinfrastruktur IT-Application oder Multimedia mit der anerkannten bE_Methode $e^{\oplus}$ einheitlich dekomponiert, zu Services modelliert und als wiederverwendbare, individualisierbare Standardleistungen im Katalog abgebildet. Dieser Katalog beinhaltet vielschichtige Informationen $\mathrm{zu}$ :

- den enthaltenen Leistungen (HW, SW, Prozessleistungen/Stunden etc.)

- den wählbaren Funktionen und den möglichen Qualitäts-Leveln

- den Herstellkosten (Stücklisten, Arbeitspläne, Lizenzen, Volumen wie z. B. Speicherbedarf etc.)

Um dabei für alle Produkte gleich vorgehen und die vielfältigen Themen gleichwertig behandeln zu können, bedarf es einheitlicher Merkmalsdefinitionen, Regelwerke und definierter Prozesse. Die Swisscom Operations definierte deshalb 10 Prinzipien für die Produktmodellierung:

1. Die Funktionen und Leistungen der Produkte stehen im Zentrum.

Der Blickwinkel der Leistungsbezüger (das WAS bzw. der Bedarf des Kunden) ist hierfür relevant. Es sollen individualisierbare Leistungen aus einer Modularisierung von Leistungselement bei gleichzeitiger Standardisierung derer Variabilität entwickelt werden, wobei die möglichst hohe Wiederverwendung von Halbfabrikaten in diesen individuell zusammengestellten Produkten das Ziel sein soll (vgl. auch Prinzip Nr. 6).

2. Koexistenz von Economy of Scope (Servicebereitstellung beim Kunden) \& Economy of Scale (individualisierter Massenfertigung).

Durch die Schaffung von Transparenz über heute bereits produzierte Leistungen und der Entkoppelung des WAS vom WIE (siehe auch - Abb. 8.7) können individuelle Produkte am 
Markt angeboten (Scope) und durch Wiederverwendung bestehender Infrastruktur trotzdem in ökonomisch interessanten Massen produziert werden (Scale).

3. Portfolios definieren die Produktverantwortung (für z.B. Plattformleistungen \& interne/externe Marktleistungen bei Organisationen).

Um den vielfältigen Bedürfnissen bzw. Sichten innerhalb der verschiedenen Einheiten gerecht zu werden, soll in der Produktverantwortung die Entkopplung von WAS und WIE gespiegelt werden. In den Commercial-Portfolios sollen verkauf- bzw. lieferbare Produkte für Endkunden (WAS), in Production-Portfolios die für die CFUs (= Customer Facing Units = Vertriebseinheiten der Swisscom) produzier- bzw. lieferbaren Leistungen bewirtschaftet werden. Um die benötigten Wertschöpfungstiefen nutzergerecht abbilden zu können, können je nach Bedarf mehrere CFUs und mehrere Production-Portfolios definiert werden.

4. End-to-End(E2E)-Governance der Servicearchitektur.

Die E2E-Governance verbindet die teilweise verschiedenen Vorgaben aus den unterschiedlichen Portfolios. Sie regelt und orchestriert entlang der ganzen Wertschöpfungskette alle relevanten Themen (definiert einheitliche Methoden und E2E-Prozesse, Begriffe, Merkmale, Regeln \& Symbole, Releases, Status etc.) und erlaubt es, die richtigen Dinge auch richtig zu tun.

5. Entkopplung Katalog \& Serviceinventar (Produkt- \& Angebot-Lebenszyklus).

Im Katalog werden die bestellbaren Leistungen (mit allen möglichen Funktionen und Ausprägungen) abgebildet, im Serviceinventar dagegen aktive Instanzen (d.h. z. B. Angebote und Vertragsentwürfe) hinterlegt (• Abb. 8.6).

6. Entkopplung von Core Service \& Service Part.

Eine durchgehende Methodik bei der Modulierung von Services und die Entkopplung zwischen der Ebene Core-Service (lieferbare Einheit) und Service Part (Halbfabrikat) schafft die Grundlage für die individualisierte Wiederverwendung von Halbfabrikaten bzw. Infrastruktur.

7. Entkopplung von Service Level (SLA) \& Operational Level (OLA) Agreements.

In der industriellen und verteilten Produktion sollen Vereinbarungen einheitlich, standardisiert und durchgängig getroffen werden, um interne und externe Kundenbeziehungen (durch SLAs) und interne Leistungserbringungsbeziehungen (durch OLAs) effizient $\mathrm{zu}$ «objektivieren» bzw. zu steuern.

8. Entkopplung von Prozessleistungen, Status \& Releases je Servicearchitektur-Ebene. Im Produktentwicklungsprozess soll für eine neue Anforderung die Phase «Setup» nur einmalig durchlaufen werden, d. h. Anpassungen werden gleichzeitig auf allen Ebenen ausgelöst. Sobald die Anforderungen aller Ebenen in «ready for service» sind, wird die Betriebsphase angestossen. Auf diese Weise erfolgt die Evolution von Services in kleinen Schritten durch verschiedene Releases und der Betrieb einer neuen Anforderung kann wiederkehrend aufgenommen bzw. in beliebig vielen Services wiederverwendet werden.

9. Entkopplung von Service Management \& Production Management.

Analog zu Prinzip Nr. 7 müssen das Production Management und das Service Management getrennt und entsprechende Rollen aufeinander abgestimmt werden. Core Services, Service Packages \& Business Services gehören dabei zum Service Management und werden mithilfe von Service Level \& Mengen Reports gesteuert und überwacht. Service Assets und Configuration Items werden im Rahmen des Production Management mithilfe von Operation Level Reports gesteuert und überwacht. 


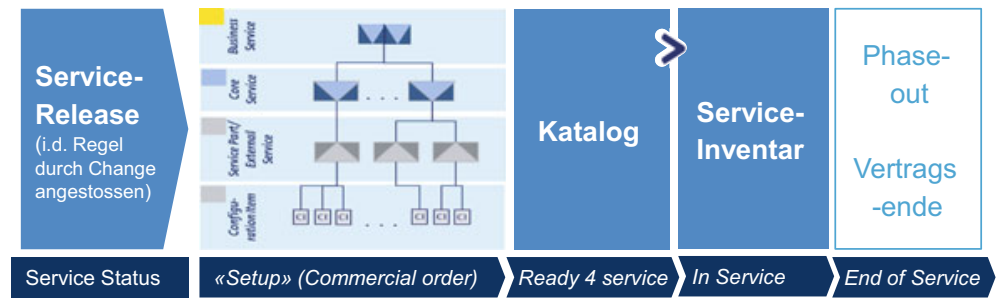

- Abb. 8.6 Services-Lebenszyklus

10. Financial, Quality \& Capacity Controlling (Mengen, Kosten, Qualität, Umsätze).

Im Bereich Service Management werden Kennzahlen zu E2E-Verfügbarkeit von Systemen und deren Performance, im Bereich Production Management Kennzahlen für die Infrastruktur- und Plattformverfügbarkeit ausgewiesen und überprüft.

Basierend auf den 10 Prinzipien wurden mithilfe der folgenden und weiteren Fragen die internen Produktmanager zu den existierenden Services befragt, darauf aufbauend eine neue Service- und Produktarchitektur abgebildet, die dekomponierten Services richtigen Ebenen zugeordnet und erfasst:

- Welcher Nutzen (Funktionen, Features) soll mit dem Produkt X beim Kunden erzeugt werden?

- Wie modular (Optionen, Varianten) muss das Produkt sein?

- Welche Service-Level-Zielwerte können vereinbart werden?

- Welche Teilleistungen können aus anderen Portfolios oder extern bezogen werden?

- Wo liegen die Verantwortungsbereiche in der bestehenden Organisation?

- Können die Service Parts wiederverwendet werden?

- Ergebnisse Phase 1 «Produktionskataloge für interne Betriebs- \& externe MarktLeistungen»

Seit April 2016 sind die neuen Kataloge - konsequent und strikt methodisch in Portfolios entlang der Wertschöpfungskette strukturiert - verfügbar. Sie bilden leistungsorientierte und ITStack-unabhängige Servicebäume, wodurch die Portfolio-übergreifende Integration von Leistungen sichergestellt wird. Durch die Baumstruktur sind auch die Leistungs- und Kosteninformationen einfach aggregierbar.

Während der Produktmodellierung stellte sich Network \& IT Operations die Frage, ob alle potenziell möglichen Ausprägungen der Leistungen und Infrastruktur (z. B. alle Typen von Betriebsservern) analysiert und abgedeckt werden sollen. Es wurde bewusst entschieden, nicht alle Details im ersten Schritt aufzunehmen, sondern schnell die Gesamtbreite des Portfolios abzudecken und allfällige Lücken im Gebrauch zu bereinigen.

Erste positive Ergebnisse stellten sich bereits während der Erstellung des Kataloges ein. So fragten die Produktmanager bereits in dieser Phase nach den standardisierten Produkten nach, was ein wichtiger Beitrag und gewünschter Effekt für die Wiederverwendung von bestehenden Leistungen ist.

॥ Die Produktkataloge liefern die Grundlage für die industrialisierte Fertigung (Andreas Hilber, Head of Process Architecture Network \& IT Operations, Swisscom AG). 


\section{- Nächste Schritte}

Die erste Projektphase («Produktkataloge für interne Betriebs- \& externe Markt-Leistungen») erreichte mit der Bereitstellung normalisierter Servicedaten den angestrebten Status, der notwendig ist, um in die zweite Projektphase (End-to-End-Prozesse zwischen Produktion und Vertrieb) überzugehen.

Im April 2016 hat Swisscom Network \& IT Operations diese zweite Phase gestartet. Im Fokus stehen die Durchgängigkeit, Standardisierung und Automatisierung der datengetriebenen End-to-End-Prozesse und die deutliche Vereinfachung der Katalognutzung für die Mitarbeitenden (interne Leistungsbezüger) durch die Realisierung einer internen Online-Shop-Lösung. Damit soll die Wiederverwendung der Katalogelemente erhöht werden.

\subsection{Fazit}

Die vorliegende Fallstudie zeigt das Vorgehen und die Erfahrungen der Swisscom Network \& IT Operations bei der Standardisierung ihrer Leistungen und der Entwicklung einer neuen Service- und Produktarchitektur. Getrieben durch die steigende Komplexität der Produkte und die wachsenden Qualitätsanforderungen der Kunden, welche die Betriebskosten erhöhen, galt es, bei gleichzeitiger Erhöhung der Qualität Kosten zu reduzieren und die Effizienz zu steigern. Inspiration holte sich Swisscom aus der herstellenden Industrie und orientierte sich an der individualisierten Massenproduktion (industrialisierten Fertigung). Um das langfristige Ziel, «leading-edge ICT Provider» zu erreichen, wurden fünf Stufen definiert, die einen Rahmen für eine Einführung in Phasen und für den Einsatz und die kontinuierliche Verbesserung dieser Methode im täglichen Betrieb bilden.

Mit der Umsetzung der ersten Phase wurde der Grundstein für die Einführung der industrialisierten Fertigung gelegt. Eine neue Produkt- und Servicearchitektur wurde entwickelt, Services dekomponiert und als wiederverwendbare, individualisierbare Standardleistungen in transparenten, homogenen und entlang der Wertschöpfungskette strukturierten Katalogen abgebildet. Dies wurde insbesondere durch die konsequente Trennung des WAS und des WIE bzw. die Entkopplung von Produkt- und Produktionssicht (- Abb. 8.7) ermöglicht und auf verschiedenen Ebenen appliziert Die dabei entstandenen normalisierten Daten bilden die Voraussetzung, um die weiteren Stufen der Transformation in Angriff nehmen zu können.

Gleichzeitig wurden Prinzipien und Methoden entwickelt, die nach der erstmaligen IstAufnahme und -Abbildung der Produkte auch weiterhin eingesetzt werden, um die Kataloge aus der Projekt- in die Betriebsphase zu überführen und ihre künftige aktive Bewirtschaftung sicherzustellen. Dadurch wird sich auch der Bereitstellungsprozess verändern, der bis dato einen starken Projektcharakter aufwies.

॥ Hard ist soft und soft ist hard - erfolgreiches Katalogmanagement setzt eine Kulturveränderung mit motivierten Mitarbeitenden voraus (Andreas Hilber, Head of Process Architecture, Network \& IT Operations, Swisscom AG).

Die Wirkung der bereits erreichten Änderungen quantifiziert Swisscom Network \& IT Operations mit einer Effizienzsteigerung von drei bis fünf Prozent. Erhöht wurde auch die Akzeptanz der internen Leistungsbezüger, die die Transparenz der Servicekomponenten sehr schätzen und diese bewusst und gezielt anwenden. Nun gilt es, diesen «Spirit» weiter zu tragen und alle Mitarbeitenden vom Nutzen des Kataloges zu überzeugen. Die wichtigsten Faktoren, die zu diesem Erfolg geführt haben, fasst $\bullet$ Abb. 8.8 zusammen. 
„Der Katalog bildet

jederzeit das verfügbare Leistungsangebot marktgerecht $a b^{\prime \prime}$

„Deutliche

Kostenreduktion und Effizienzsteigerung bei gleichzeitiger Erhöhung von «Time to Market und Qualität"

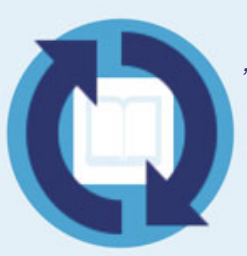

„Anpassungen des Leistungsangebots der

Das ermöglicht Produktion erfolgt nur nach Abwägung von Aufwand \& Nutzen"
„Halbfabrikate können effektiv wiederverwendet und Komplexität reduziert werden

Das bedingt

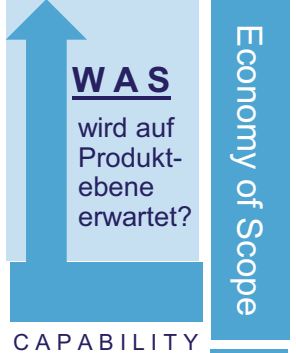

CAPABILITY

- Abb. 8.7 Gesamtübersicht Ziele, Vorgehen, Lösungsansatz

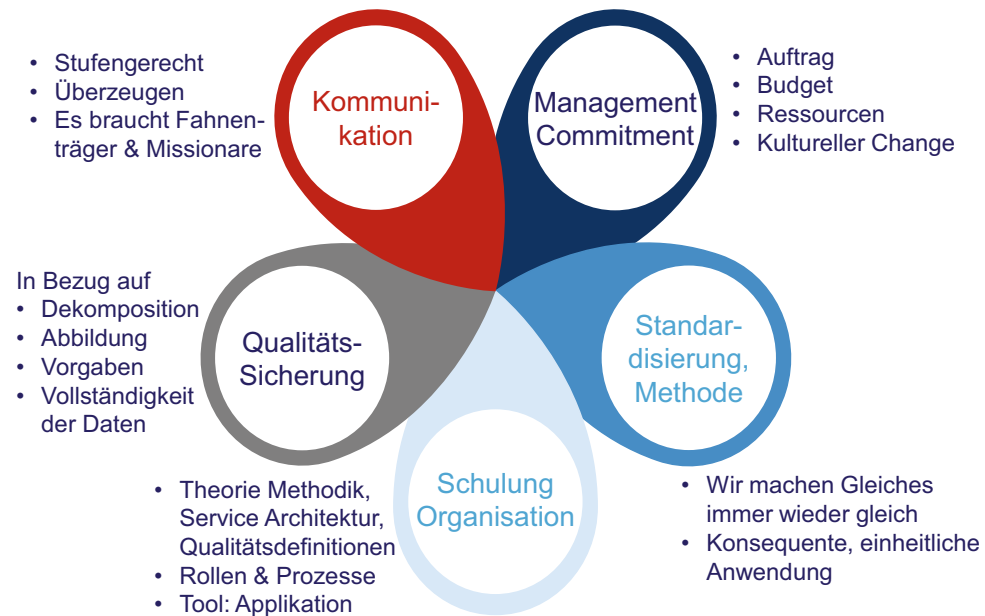

- Abb. 8.8 Erfolgsfaktoren

- Einbettung in das Studienframework - Kundennutzen durch digitale Transformation?

Im Unterschied zu Unternehmen in manch anderen Branchen, die über Relevanz und Fokus der Digitalisierung noch nachdenken, ist sie für Swisscom Kerngeschäft. Dem Betrieb einer Netzwerk- und IT-Infrastruktur, deren Zweck es ist, Geschäfts- und Privatkunden, Partnern und Mitarbeitenden

«Das Beste in der vernetzten Welt - immer und überall zu bieten» (Swisscom AG 2015), kommt dabei zweifelsfrei eine Schlüsselrolle zu. In diesem Sinne stellt die Swisscom-Strategie 2020 die Ziele «beste Erlebnisse bieten» und «beste Infrastruktur bauen» auf eine Ebene. Das eine ist ohne das andere nicht möglich. Die vorliegende Fallstudie macht diesen Wirkungszusammenhang deutlich. Sie zeigt auf, dass ein effizienter und hochstandardisierter IT-Betrieb 


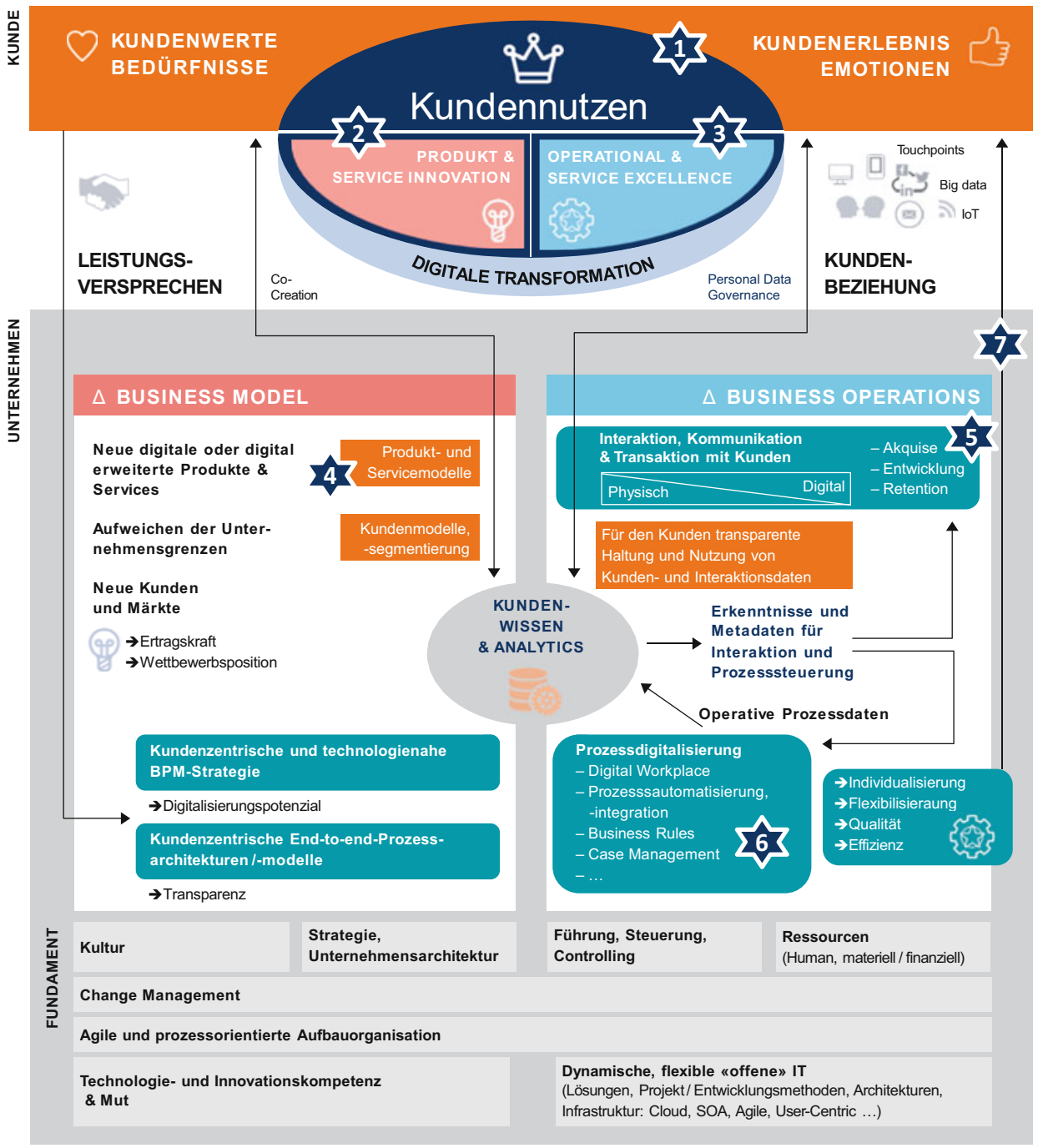

- Abb. 8.9 Swisscom Fallstudie - Kernaspekte im Kontext des Studienframeworks

nicht nur für Operational \& Service Excellence steht, sondern sich auch auf die Fähigkeit, Produkte und Services rasch zu innovieren und auf das Kundenerlebnis auswirkt.

Die Fallstudie deckt somit ein breites Spektrum an Aspekten aus dem Studienframework ab, die in - Abb. 8.9 gekennzeichnet und nachfolgend ausgeführt sind. 


\section{Mit welcher Zielsetzung und mit welcher Wirkung wird digital transformiert? Welcher Kun- dennutzen wird angestrebt bzw. wurde bereits realisiert?}

\section{Kundennutzen: Transparenz \& Zugänglichkeit}

Die Fallstudie zeigt, dass Prinzipien wie Konsistenz und Einfachheit für Swisscom nicht nur für die Gestaltung von Endnutzerdiensten gilt, sondern auch für den IT-Betrieb. Die Reduktion von Komplexität macht die Infrastruktur nicht nur für Network \& IT Operations beherrschbarer und effizienter, sondern macht sie auch transparenter und begreifbarer für die Leistungsbezieher. Verfügbare Produkte, deren Leistungsumfang und Herstellkosten sind aus den Katalogen rasch ersichtlich und auswählbar.

\section{Produkt- \& Service Innovation: Time-to-Market}

Standardisierung und Zugänglichkeit bewirken, dass Leistungen, aufgrund ihrer Eigenschaften schneller abgerufen werden können. Die Entwicklung neuer Produkte und Services wird beschleunigt, da bestehende Komponenten wiederverwendet werden können, anstatt bei jedem Bedarf Anforderungen aufs Neue zu spezifizieren.

\section{Operational Service Excellence: Economies of scale \& scope}

Durch die Wiederverwendbarkeit nach dem Vorbild der Halbfabrikate in der industriellen Fertigung werden Skaleneffekte erzielt (drei bis fünf Prozent realisierte Effizienzsteigerung). Leistungsbreite und Leistungstiefe sind aus Kundensicht erhöht, da die Produktkataloge ein grosses Spektrum an individuell kombinierbaren Produkten integrieren.

\section{Was wurde bzw. wird digital transformiert?}

\section{Business Model: «Produkt- und Service-Modelle»}

Swisscom Network \& IT Operations hat sich mit den angebotenen Produkten und Dienstleistungen auseinandergesetzt und in einer Produkt- und Servicearchitektur neu strukturiert. Grundprinzip der Produktmodellierung ist die Frage «Was erhält der interne oder externe Kunde an nutzbaren Leistungen?» («Know-Your-Product»).

\section{$\mathbf{5}$ Business Operations}

Mit dem beschriebenen 5-Stufen-Konzept werden die kritischen Geschäftsfähigkeiten, die Swisscom Network \& IT Operations als «leading-edge ICT Betrieb mit internationaler Reputation» anstrebt (Produktkataloge, durchgängige Prozesse, Serviceinventar, Service Engine etc.), definiert, schrittweise umgesetzt und kontinuierlich verbessert («Know-Your-Capabilities»)

Wie und wodurch wird transformiert? (Technologieeinsatz, Elemente der Prozessdigitalisierung)

\section{$\widehat{6}$ Produktdaten und Automatisierung}

Mit den Produktkatalogen, die die Services (Hardware, Software und Prozessleistungen) dekomponiert, standardisiert und einheitlich abbildet, wurde die Grundlage für automatisierte und datengetriebene Serviceprozesse geschaffen. Der nächste Schritt - Automatisierung durchgängiger Prozesse - wurde gestartet.

\section{Online Shop}

Die Interaktion mit Swisscom-internen Leistungsbezügern wird in Phase 2 durch die Bereitstellung der Kataloge in einem internen Online-Shop realisiert. Dies ist ein erster Schritt, um automatisierte Produktion in durchgängige End-to-End-Prozesse zu integrieren. 


\section{Literatur}

Springer Gabler Verlag (ohne Jahr) (Hrsg.). Gabler Wirtschaftslexikon, Stichwort: Industrialisierung. http:// wirtschaftslexikon.gabler.de/Definition/industrialisierung.html. Abgerufen am 16.6.2016.

Swisscom AG (2015). Unternehmensstrategie «Swisscom 2020». https://www.swisscom.ch/de/about/ unternehmen/strategie.html. Abgerufen am 16.6.2016.

Open Access Dieses Kapitel wird unter der Creative Commons Namensnennung 4.0 International Lizenz (http://creativecommons.org/licenses/by/4.0/deed.de) veröffentlicht, welche die Nutzung, Vervielfältigung, Bearbeitung, Verbreitung und Wiedergabe in jeglichem Medium und Format erlaubt, sofern Sie den/die ursprünglichen Autor(en) und die Quelle ordnungsgemäß nennen, einen Link zur Creative Commons Lizenz beifügen und angeben, ob Änderungen vorgenommen wurden.

Die in diesem Kapitel enthaltenen Bilder und sonstiges Drittmaterial unterliegen ebenfalls der genannten Creative Commons Lizenz, sofern sich aus der Abbildungslegende nichts anderes ergibt. Sofern das betreffende Material nicht unter der genannten Creative Commons Lizenz steht und die betreffende Handlung nicht nach gesetzlichen Vorschriften erlaubt ist, ist für die oben aufgeführten Weiterverwendungen des Materials die Einwilligung des jeweiligen Rechteinhabers einzuholen.

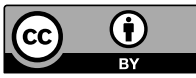

